\title{
Potential use cases for the development of an electronic health facility registry in Nigeria: Key informant's perspectives
}

\author{
Olusesan Ayodeji Makinde ${ }^{1,2}$, Aderemi Azeez ${ }^{3}$, Wura Adebayo ${ }^{3}$
}

1. Viable Knowledge Masters, Abuja, Nigeria

2. Demography and Population Studies Program, Schools of Public Health and Social Sciences, University of the Witwatersrand, Johannesburg, South Africa

3. Department of Health Planning, Research and Statistics, Federal Ministry of Health, Abuja, Nigeria

\begin{abstract}
Background: Master facility lists (MFL) maintain an important standard (unique identifier) in country health information systems that will aid integration and interoperability of multiple health facility based data sources. However, this standard is not readily available in several low and middle income countries where reliable data is most needed for efficient planning. The World Health Organization in 2012 drew up guidelines for the creation of MFLs in countries but this guideline still requires domestication and process modeling for each country adopting it. Nigeria in 2013 published a paper-based MFL directory which it hopes to migrate to an electronic MFL registry for use across the country.

Objective: To identify the use cases of importance in the development of an electronic health facility registry to manage the MFL compiled in Nigeria.

Methods: Potential use cases for the health facility registry were identified through consultations with key informants at the Federal Ministry of Health. These will serve as input into an electronic MFL registry development effort.

Results: The use cases identified include: new health facility is created, update of status of health facility, close-out, relocation, new information available, delete and management of multi-branch health facility.

Conclusion: Development of an application for the management of MFLs requires proper architectural analysis of the manifestations that can befall a health facility through its lifecycle. A MFL electronic registry will be invaluable to manage health facility data and will aid the integration and interoperability of health facility information systems.
\end{abstract}

Keywords: Health Facilities; Health Information Exchange; Master Facility List; Pubic Health Informatics; Registries; Standards

Correspondence: Email- sesmak@gmail.com

DOI: $10.5210 /$ ojphi.v8i2.6350

Copyright @2016 the author(s)

This is an Open Access article. Authors own copyright of their articles appearing in the Online Journal of Public Health Informatics. Readers may copy articles without permission of the copyright owner(s), as long as the author and OJPHI are acknowledged in the copy and the copy is used for educational, not-for-profit purposes. 


\section{Introduction}

Health information systems (HIS) have been widely described as the foundation of public health, responsible for driving evidence-based decisions (1). However, their ability to drive the health system has been sub-optimal in several countries (2-4). This deficiency has been more prominent in low and middle income countries (LMIC) where evidence-based resource allocation is most needed but reliable data is hardly available. The unavailability of reliable health data arises as a result of lack of processes and systems, poor human resource capacity and the huge cost attached to data management $(3,5,6)$. Also, the structure of paper-based HIS that characterize health systems in LMIC are inefficient (5). To address these shortfalls, several developing countries have begun deploying electronic applications for the management of their routine health data (7). However, these deployments are challenged by the unavailability of standards that will facilitate data exchange $(8,9)$. One major benefit of health Information Technology (IT) is the ability to gather data on a single health facility from multiple points of generation and use these data for multi-level decision making (10). Unfortunately, this is only possible when these information systems can be linked and exchange data.

Identification of health facilities across multiple information systems can pose a big challenge to the success of this endeavor (10). This arises as health facilities can change names or there could be more than one health facility with the same name, thus making the name of the health facility undesirable as a unique identifier across systems, a necessity for integration and interoperability of different information systems which house health facility data. Several other technical, motivational, economic, political ethical and legal barriers have been implicated as contributing to the inability of HIS to exchange data $(11,12)$. To address the identity challenge for health facilities, the World Health Organization (WHO) in 2012, developed guidelines for the creation of Master Facility Lists (MFL) for countries (13). According to WHO, "a MFL is a complete listing of health facilities in a country (both public and private) and is comprised of a set of identification items for each facility (signature domain) and basic information on the service capacity of each facility (service domain)" (13). The country MFLs will serve as a repository for the allocation and maintenance of unique identifiers, the standard that will facilitate linking of health facility data sources thereby aiding integration and interoperability of these systems (14).

Since health facilities are continuously built and some close out, the processes to update the MFL are a necessary step in making the MFL continuously relevant and useful. In an earlier paper from Nigeria, absence of processes and an information system to manage the MFL were identified as major limitations which threatened the success and usefulness of the MFL compiled (10). Handling this gap requires extensive analysis of the actions that can befall a health facility in the MFL over time and setting up a detailed registry to respond to all the scenarios. The possibility of implementing a MFL management registry via paper-based processes is complex and it is not guaranteed that it will be achievable when there are several players involved across large geographic areas. This has affected the ability of the MFL developed in Nigeria to be kept up-to-date since established. In addition, since routine health information systems (RHIS) are being moved to electronic platforms (10), it will be cumbersome to ensure that paper based steps are continuously synchronized with applications using the MFL products electronically. The chance for moving the management of the MFL to an electronic registry necessitates detailed planning to ensure its success. 
In this paper, we describe the potential scenarios that can befall a health facility within a MFL which will serve as inputs when developing an electronic registry to manage the MFL. Our focus is on Nigeria where this planning activity took place.

\section{Methods}

In 2013, Nigeria published a MFL booklet directory which allocated unique identifiers to all the health facilities within the country (15). The process for achieving this and the parameters captured in the MFL have been described by the Federal Ministry of Health (FMOH) and partners elsewhere (10). Nigeria is a Federation and the governance structure in the country is three tiered: the federal government is the national government and gives policy directions, the state governments (36 states and the Federal Capital Territory) oversee affairs at the states and localize and implement policies at a lower level, while the local government is the closest level of governance to the people and implements activities and policies at a lower level than the states. The compilation of the MFL had been carried out by the FMOH working with the states to collate the data on all the hospitals and clinics in their various domains to come up with the national MFL following the allocation of a unique identifier to each health facility. Health facility registration is carried out at the state ministries of health and thus, 37 different registries are required to manage the national health facility records. The parameters captured in this directory include the name of the health facility, the state of location, the local government area of location within the state, the ward of location within the local government, the ownership of the health facility (Private or Public), the level of care provided (Primary, Secondary or Tertiary) and a unique provider identifier. This unique identifier was generated through an intelligent coding system that concatenates values allocated based on the described parameters.

Consultations were held with key personnel in the FMOH in Nigeria between 2013 and 2014 to document the potential scenarios that can befall a health facility through its life cycle. This information is important for planning when developing an electronic registry application to manage the MFL. These key personnel were the people who initiated the compilation of the MFL earlier, and were the most knowledgeable about the national MFL within the country. The consultations were both one-on-one and in groups to jointly outline the important processes necessary for an electronic health facility registry. The group discussions served as an opportunity to validate and agree on the most important first steps in developing the system. Documentation was completed by the lead author during these consultative sessions.

The identified pathways were presented and further discussed in follow up sessions before a list of processes was arrived at. These scenarios have not been scored or arranged by any level of importance.

\section{Potential Use Cases/ Results}

The scenarios presented in this paper are a first step which though, not believed to be exhaustive, are needed to be planned for in the development and roll out of an electronic registry for the management of the MFL. This can subsequently be built upon as new scenarios which were initially unplanned for emerge as the application is put to use. As a base, the MFL compiled will be uploaded into the system and will be the starting point for any follow up updates. 
The identified scenarios are new health facility creation, update information on an already listed health facility/ change of status, close out, relocation of health facility, additional information available, delete a health facility and management of multi-branch health facility. These are further elaborated upon in the next section.

\section{New health facility}

Continuously, health facilities are being established in the country and once established need to be issued unique identifiers and listed in the MFL. Also, other necessary information on the health facility needs to be captured and archived. The registry must be able to accept the creation of a new health facility in the system and maintain a unique identifier for the facility. Though not yet appropriately defined, the need for this step to be completed by an accreditation agency that has certified the health facility, properly equipped and ready to provide services in a specific category (primary, secondary or tertiary) was identified. A process must be developed to facilitate information transfer from the accreditation agency to the MFL managers or a portal which allows this accreditation agency to log this information directly into the electronic registry. The information stored should include a date of approval and the authority that granted the health facility an operational right. This is particularly important if there are multiple organizations that can grant operational rights within the country.

\section{Update information on a health facilityl Change of status}

A health facility may be upgraded from primary to secondary, secondary to tertiary or downgraded if the criteria for accreditation change or the health facility fails to meet the status for accreditation during a re-evaluation exercise. This re-evaluation exercise might need to be conducted at a specified interval to continuously check that health facilities are maintaining agreed standards and to assure the quality of care provided. Also, the ownership of a health facility can change from private to public or vice-versa, if it is bought over or bequeathed by a former private owner to the government. The MFL registry must permit the change of status of a health facility and must be able to provide the status by any date queried in case the status has changed. This must be factored into the design of the MFL registry application.

\section{Close out}

Health facilities can close out for various reasons. For example, a one-man practice may have to shut down if the proprietor dies and there is no one else licensed to maintain the health facility accreditation. However, the details of the health facility must remain archived including when the close-out status was achieved and possibly, the circumstances that led to this close-out. In this situation, the system must be designed such that the health facility does not contribute any further, to the statistics of health facilities while its information is still retrievable on a need basis. The status of health facilities (active or dormant) might be determined during an annual licensing routine or when evaluators visit the health facility for quality monitoring. Thus, the process for which information from these follow up assessments feed into the MFL registry should be properly established. 


\section{Relocation of health facility}

In Nigeria, the standard process for the generation of a unique health facility identifier incorporates the local government area and state of location of the health facility $(10,15)$. Thus, if a health facility moves to a new local government area or state, it nullifies the existing unique identifier and a new one must be allotted. If the relocation is still within the same local government, the process for updating the location information should be properly designed into the system with comments on the reason for the change. In this situation, the health facility retains its unique identifier.

\section{Additional information available}

At the baseline of the collation of a MFL, it is unlikely that all the important data on each health facility will be available. This is particularly important in Nigeria as the compilation of the MFL recently completed had fewer than 10 parameters available for each health facility as previously described (10). As such, the MFL registry should be scalable and there should be opportunity to continuously add data on each health facility so that the records can be built up over time. Several health facility assessments are conducted in Nigeria for various reasons and by different parties in the country and these efforts can be leveraged to improve the completeness of the data in the national MFL.

\section{Delete a health facility}

Deleting will be a restricted action which would be sanctioned only when there has been an approval for this process to move ahead by the authorities that govern the management of the MFL. Situations for which the deletion of a health facility can be sanctioned will include when a health facility has been wrongly created in the system and there is no associated data to the health facility or the data has been migrated elsewhere. In this case, the system should still maintain a deleted files log that can be retrieved.

\section{Multi-branch health facility}

Health facilities may operate in more than one physical location and this raises issues on the management of these affiliated health facilities in the MFL registry. Each site will need to be accredited independently and granted an independent unique identifier in the MFL. Nigeria uses an intelligent coding system in the generation of the unique identifier which carries some information on where the health facility is located. Attempting to use the same unique identifier for more than one health facility site will result in an error of attribution in the system. As such, health facilities affiliated with a parent will be treated as independent health facilities and will need to be issued independent unique identifiers.

While handling multi-facility identification is important, this is not a specific process that needs to be programmed into the system but is an important governance issue that was repeatedly echoed during the consultative meetings. 


\section{Discussion}

The continued usefulness of a MFL requires that it is updated and used in the knowledge generation process. The MFL registry will maintain the unique identifier of health facilities which is a technical standard in enhancing integration and interoperability of HIS, unavailability of which can limit the gains of a country HIS. It is a necessary standard for health information exchanges to function and link different health facility based data sources that are useful in health planning. The interconnection of these different data sources is a significant first step to routine data use for public health surveillance (16). A recent systematic review identified poor availability and use of technical standards as one of the barriers to data sharing across the world (11). Metadata and standards are lifelines that can always help to achieve information system continuity. Their absence or unreliability can be a limitation to the success of integration and interoperability of sub-systems. Thus, the MFL registry as the harbor of a technical standard will be a major hub for linking multiple health facility data sources in Nigeria as the country continues to adopt IT in the management of health facility data.

Recognizing the complexity of MFL management and the importance of keeping an up-to-date MFL registry for developing countries, two prominent international initiatives (Facility Registry and the Open Health Information Exchange) have been launched which intend providing open source applications for managing health facility registries and enhancing the exchange of health data between information systems that house health facility data $(17,18)$. However, these efforts need to be fed with the processes that are important for managing MFLs. Most MFLs in different countries will have some basic similar processes. However, there will be some specific considerations that are necessary for different countries which need to be customized to suit the country’s specific needs.

Since the status of a health facility can change over time, the system must be able to store the information longitudinally. This will facilitate the ease of determining the status of a health facility at any point retrospectively. To ascertain that the status of a health facility is always up to date in the MFL electronic registry, it might be necessary to periodically assess the status of health facilities in a local government or state. An opportunity to achieve this can be through an annual license or recertification exercise. This, besides making the MFL reliable, will further ensure an accurate denominator statistic when the number of active health facilities is required for calculating routine health indicators. The responsibility to carry out these routine assessments must be incorporated in the health facility accreditation organization or unit in the country, with the outcome of the assessment fed to the registry.

The recently assented Nigerian National Health Act of 2015 in Part II (Health Establishments and Technologies), sections 12-19 further provides legal credence for the better coordination of health facility registries and accreditation organizations in states (19). This section of the act necessitates proper classification of all health establishments, with definition of their role within the national health system along with their installed capacity. Based on an assessment, a certificate of establishment will be issued to the health facility that will specify the category of services for which the establishment is licensed to operate. In event that the establishment is interested in scaling services, a reevaluation will be necessary based on a new set of criteria. This section of the act also prescribes penalties for defaulters. As this new National Health Act begins 
to be implemented, the role of the MFL registry will become of greater importance for managing these important data and providing the knowledge to the government and the public.

Beside the processes in the information system, the human steps for managing the data generation process are equally important. This will include outlining the organizations involved and detailed step by step processes to ensure quality is maintained at each registration point which are in Nigeria's 36 states and the Federal Capital Territory.

\section{Limitations}

With the rise in the use of computers and information systems in healthcare delivery and the concomitant expansion of global health indicators, there has been undue emphasis on hardware and software in health information systems without similar effort on the people and processes that will make the systems work effectively (20). This is a major threat to the success of the MFL registry development endeavor in Nigeria and thus there is an increased need to educate and advocate to major stakeholders and decision makers on the need to address the HIS holistically including the establishment and empowerment of governance systems.

Furthermore, the procedures identified herein are not exhaustive and will require additional investigation and scale up as the application is put to use. The challenge of sustainable funding for health information exchanges (HIEs) which has been identified as a major threat to the sustainability of HIEs in developed countries is also a threat in developing countries (12). As such, models that will provide for sustainable financing of the systems should be considered as developing countries continue to adopt applications that will facilitate integration and interoperability of their HIS.

\section{Conclusion}

The MFL registry is an important platform for managing and maintaining the unique identifier for health facilities, a necessary standard that will aid the integration and interoperability of several health facility data sources. Development of an application to manage the MFL must take into consideration several potential scenarios that can befall a health facility through its life course. The MFL registry will provide an appropriate platform for managing the pronouncements of the Nigerian National Health Act of 2015 on health establishments. The MFL registry to be developed should be scalable to capture new use cases as new requirements emerge.

\section{Acknowledgements}

The efforts of the staff of the Monitoring and Evaluation division of the Federal Ministry of Health and other participants during the consultations are appreciated.

\section{Conflicts of Interest}

OAM worked for MEASURE Evaluation between April 2013 and February 2015 which received funding from the United States Agency for International Development to support the Government of Nigeria on health information systems strengthening. 


\section{References}

1. Abouzahr C, Boerma T. Health information systems: the foundations of public health. Bull World Health Organ [Internet]. 2005 Aug [cited 2013 Nov 30];83(8):578-83. Available from: http://www.ncbi.nlm.nih.gov/pmc/articles/PMC2626318/

2. Amouzou A, Kachaka W, Banda B, Chimzimu M, Hill K, et al. Monitoring child survival in "real time" using routine health facility records: results from Malawi. Trop Med Int Health [Internet]. 2013 Oct [cited 2013 Dec 2];18(10):1231-9. Available from: http://www.ncbi.nlm.nih.gov/pmc/articles/PMC3787785/

3. Aqil A, Lippeveld T, Hozumi D. PRISM framework: a paradigm shift for designing, strengthening and evaluating routine health information systems. Health Policy Plan [Internet]. 2009 May [cited 2014 Mar 6];24(3):217-28. Available from: http://www.ncbi.nlm.nih.gov/pmc/articles/PMC2670976/

4. Aung E, Whittaker M. Preparing routine health information systems for immediate health responses to disasters. Health Policy Plan [Internet]. 2013 Aug [cited 2013 Dec 2];28(5):495-507. Available from: http://www.ncbi.nlm.nih.gov/pmc/articles/PMC3743306/

5. World Health Organization. Framework and standards for country health information systems [Internet]. 2008 [cited 2015 Nov 4]. Available from: http://apps.who.int/iris/handle/10665/43872

6. Makinde OA, Mami MI, Oweghoro BM, Oyediran KA, Mullen S. 2016. Investing in health information management: The right people, in the right place, at the right time [Internet]. HIM J. 45(2), 90-96. http://him.sagepub.com/content/45/2/90. PubMed

7. Karuri J, Waiganjo P, Orwa D. Implementing a Web-based Routine Health Information System in Kenya: Factors Affecting Acceptance and Use. 2014 Sep [cited 2014 Nov 12];3(9). Available from: http://www.ijsr.net/archive/v3i9/U0VQMTQ0OTg=.pdf

8. Park Y-T, Atalag K. Current National Approach to Healthcare ICT Standardization: Focus on Progress in New Zealand. Healthc Inform Res [Internet]. 2015 Jul [cited 2015 Nov 1];21(3):144-51. Available from: http://www.ncbi.nlm.nih.gov/pmc/articles/PMC4532838/

9. Board of Directors of the American Medical Informatics Association. Standards for Medical Identifiers, Codes, and Messages Needed to Create an Efficient Computer-Stored Medical Record. J Am Med Inform Assoc [Internet]. 1994 Jan 1 [cited 2013 Dec 15];1(1):1-7. Available from: http://jamia.bmj.com/content/1/1/1

10. Makinde OA, Azeez A, Bamidele S, Oyemakinde A, Oyediran KA, et al. Development of a Master Health Facility List in Nigeria. Online J Public Health Inform [Internet]. 2014 Oct 16 [cited 2014 Nov 27];6(2). Available from: http://www.ncbi.nlm.nih.gov/pmc/articles/PMC4235326/

11. van Panhuis WG, Paul P, Emerson C, Grefenstette J, Wilder R, et al. A systematic review of barriers to data sharing in public health. BMC Public Health [Internet]. 2014 Nov [cited 
2014 Nov 9];14(1):1144. Available from: http://www.biomedcentral.com/14712458/14/1144/

12. Akhlaq A, McKinstry B, Muhammad KB, Sheikh A. Barriers and facilitators to health information exchange in low- and middle-income country settings: a systematic review. Health Policy Plan [Internet]. 2016 May 16 [cited 2016 May 21]; Available from: http://0heapol.oxfordjournals.org.innopac.wits.ac.za/content/early/2016/05/14/heapol.czw056

13. World Health Organization. Creating a Master Health Facility List [Internet]. 2012 Mar [cited 2014 Jan 27]. Available from: http://www.who.int/healthinfo/systems/WHO_CreatingMFL_draft.pdf

14. Rose-Wood A, Heard N, Thermidor R, Chan J, Joseph F, et al. Development and use of a master health facility list: Haiti's experience during the 2010 earthquake response. Glob Health Sci Pract [Internet]. 2014 Aug 5 [cited 2014 Aug 6];ghs1400029. Available from: http://www.ghspjournal.org/content/early/2014/08/04/GHSP-D-14-00029

15. Federal Ministry of Health Nigeria. A directory of health facilities in Nigeria. Abuja, Nigeria: Federal Ministry of Health Nigeria; 2013.

16. Mirza N, Reynolds TL, Coletta M, Suda K, Soyiri I, et al. Steps to a Sustainable Public Health Surveillance Enterprise. Online J Public Health Inform [Internet]. 2013 Jun 30 [cited 2014 Jan 26];5(2). Available from: http://journals.uic.edu/ojs/index.php/ojphi/article/view/4703

17. Open Health Information Exchange. OpenHIE Community Wiki [Internet]. [cited 2014 Nov 13]. Available from: https://wiki.ohie.org/display/documents/Home

18. Facility Registry API [Internet]. [cited 2014 Nov 13]. Available from: http://facilityregistry.org/\#about

19. Federal Government of Nigeria. National Health Act. Federal Government of Nigeria; 2015.

20. Thomas JC, Silvestre E, Salentine S, Reynolds H, Smith J. 2016. What systems are essential to achieving the sustainable development goals and what will it take to marshal them? Health Policy Plan. PubMed 